\title{
A comparison of the effect of genetic improvement and seed source and seedling seed orchard variables on progeny growth in Eucalyptus nitens in South Africa
}

Tammy-L Swain ${ }^{1}$, Steve D Verryn ${ }^{2}$ and Mark D Laing ${ }^{3}$

${ }^{1}$ Institute for Commercial Forestry Research, P.O. Box 100281, Scottsville, Pietermaritzburg 3209, South Africa.

${ }^{2}$ Forestry and Agricultural Biotechnology Institute (FABI), University of Pretoria, Private Bag X20, Hatfield, Pretoria 0028, South Africa and Creation Breeding Innovations, 75 Kafue St, Lynnwood Glen, 0081, South Africa.

3 School of Agricultural Earth and Environmental Sciences (SAEES), University of KwaZulu-Natal, PO Box 375, Pietermaritzburg, 3201, South Africa.

* Author for correspondence: T-L Swain

Telephone: +27333862314

Fax: +27333868905

Tammy.Swain@icfr.ukzn.ac.za

[Published as:

Swain T-L, Verryn SD, Laing MD. 2013. A comparison of the effect of genetic improvement and seed source and seedling seed orchard variables on progeny growth in Eucalyptus nitens in South Africa. Tree Genetics and Genomes DOI 100.1007/s11295-013-0593-0. 9(3): 767-778.] 


\begin{abstract}
Eucalyptus nitens is an important forestry species grown for pulp and paper production in the temperate, summer rainfall regions of South Africa. A tree improvement programme has been ongoing at the Institute for Commercial Forestry Research for two decades, but genetic improvement in the species has been slow due to delayed and infrequent flowering and seed production. Three trials were established to firstly, quantify the gains that have been made in the first generation of improvement in the breeding programme; and secondly, establish whether a number of seed source and orchard variables influence the performance of the progeny. These variables are: the amount of flowering trees in the seed orchard, year of seed collection, seed orchard origin and composition of seed orchard seed bulks. Diameter at breast height and tree height were measured in the trials at between 87 and 97 months after establishment and timber volumes and survival were calculated. Improved seed orchard bulks performed significantly better $(p<0.01)$ than unimproved controls in the field trials. Genetic gains ranging from 23.2 to $164.8 \mathrm{~m}^{3} \mathrm{ha}^{-1}$ were observed over the unimproved commercial seed. There were significant differences $(p<0.01)$ in progeny growth between the levels of seed orchard flowering, with higher levels of flowering $(\geq 40 \%)$ producing substantially greater progeny growth than lower flowering levels $(\leq 20 \%)$. The seed orchard had no effect on progeny growth in this trial series. This suggests that seed collected from any of the four seed orchards tested will produce trees with significant improvement in growth.
\end{abstract}

\title{
Keywords
}

E. nitens, genetic gain, tree improvement, breeding, flowering levels, seed bulk composition 


\section{INTRODUCTION}

Eucalyptus nitens remains one of the most important commercial cold tolerant eucalypt (CTE) species currently grown for pulp and paper production in the summer rainfall regions of South Africa. Significant variation exists among the provenances grown in South Africa for growth (Swain et al. 1998; Gardner et al. 2003) and drought (Darrow 1996; Gardner 2001), frost and cold tolerance (Gardner 2001; Swain 2001); timing and abundance of flowering (Carlson et al. 2000; Jones 2002; Gardner and Bertling 2005); seed production (Swain and Chiappero 1998; Jones 2002) and pulping properties (Clarke 2000). This makes the species ideally suited to genetic improvement.

In South Africa, E. nitens grows optimally where the mean annual temperature (MAT) is greater than $14^{\circ} \mathrm{C}$ and less than $16^{\circ} \mathrm{C}$ (Swain and Gardner 2003). The species is classified as frost tolerant, but is not as hardy as Eucalyptus macarthurii (Darrow 1994; 1996), and is recognised as one of the most snow hardy of the CTEs grown in South Africa (Gardner and Swain 1996; Kunz and Gardner 2001). Currently, there is no alternative commercial species to E. nitens for sites prone to moderate frost and heavy snows.

The E. nitens populations grown commercially in South Africa originate from several provenances in New South Wales (NSW) in Australia, as provenance trials have shown that the material from Victoria in Australia does not perform well in the summer rainfall regions of South Africa (Swain et al. 1998). A breeding programme for E. nitens has been ongoing since the early 1980s, when the Institute for Commercial Forestry Research (ICFR) took over a series of provenance/progeny trials from the South African Department of Forestry. These trials tested a range of seedlots and provenances imported from Australia, with additional trials being established by the ICFR to assess new Australian seed imports at the end of the 1980s (Swain et al. 1998).

As vegetative propagation is difficult in E. nitens (de Little et al. 1992; Moncur 1998), open-pollinated seed orchards have been established for the production of improved seed. The reticent or shy flowering of E. nitens (Gardner 2003) has hindered the breeding programme and the production of improved seed, for plantation establishment. Globally, the species is known as a light and infrequent flowerer and produces small seed crops (Pound et al. 2003). In South Africa, the species often only becomes reproductively mature at 10 to 15 years of age if grown in a plantation situation (Eldridge et al. 1993; Gardner 2003) and requires winter chilling, or hormonal treatments to replace the chilling, if flowering is to occur earlier (Gardner and Bertling 2005). The use of open-pollinated seed orchards to turn over generations in conventional breeding is therefore slow and difficult, and can result in inconsistent commercial seed production. Shy flowering may also affect realised or actual gain, in that only certain families may be

contributing as pollen parents, potentially causing differences from predicted gain. On the contrary, if different or additional families start flowering with each advancing year, gain may vary significantly on an annual basis. The mixed mating system of E. nitens, where outcrossing is preferential but selfing is not uncommon (Griffin et al. 1987; Sedgley et al. 1989), in conjunction with the erratic flowering of the species, may result in the open-pollinated seed orchards failing to produce consistently high quality seed. 
A series of genetic gain trials was established in 2001, firstly, to quantify the gain that has been made in the first generation of improvement in E. nitens and, secondly, to establish whether there is any relationship between level of flowering in an orchard, family composition of the seed orchard bulk, the seedling seed orchard and the genetic gain in progeny derived from the ICFR's E. nitens advanced generation seedling seed orchards.

\section{MATERIAL AND METHODS}

Three genetic gain trials were established on temperate sites in KwaZulu-Natal (KZN) and Mpumalanga (MPU) in South Africa early in 2001, i.e. Balgowan, Amsterdam and Lothair. Details of the trial sites and trial designs are included in Table 1. All trials were planted at 1667 stems per hectare stocking $(2 \times 3 \mathrm{~m})$, with four replicates of treatments or entries in square plots of $5 \times 5$ trees, and only the inner 9 trees $(3 \times 3)$ being measured in order to exclude inter-treatment/entry competition effects. Twenty-five to 28 entries, details of which are in Table 2, were included in the trials. The improved material originated from four ICFR seedling seed orchards, i.e. Amsterdam, Helvetia, Jaglust and Jessievale. These were former provenance/progeny trials that were thinned to seed orchards using a $30 \%$ roguing of poor families and a thinning to the best tree per plot of remaining families. After roguing, there were only three common families across all four of these seed orchards which could potentially act as pollen parents, and an additional six that were common to three of the orchards.

Table 1 Site and trial design details of three E. nitens genetic gain trials in South Africa

\begin{tabular}{|c|c|c|c|c|c|c|c|c|c|}
\hline $\begin{array}{c}\text { Plantation, } \\
\text { Province }\end{array}$ & $\begin{array}{c}\text { Date } \\
\text { Planted }\end{array}$ & $\begin{array}{c}\text { Latitude } \\
{ }^{\circ}(\mathrm{S})\end{array}$ & $\begin{array}{c}\text { Longitude } \\
{ }^{\circ}(\mathrm{E})\end{array}$ & $\begin{array}{l}\text { Altitude } \\
\text { (m a.s.l.) }\end{array}$ & $\begin{array}{l}\text { MAP }^{a} \\
(\mathbf{m m})\end{array}$ & $\begin{array}{c}\mathbf{M A T}^{\mathbf{b}} \\
\left({ }^{\mathbf{o}} \mathrm{C}\right)\end{array}$ & $\begin{array}{l}\text { Soil depth } \\
\quad(\mathbf{m m})\end{array}$ & $\begin{array}{l}\text { No. of } \\
\text { entries }\end{array}$ & Design \\
\hline Balgowan, KZN ${ }^{\mathrm{c}}$ & $05 / 02 / 01$ & -29.4044 & 30.02417 & 1498 & 1002 & 15.3 & $1000-1200$ & 28 & $5 \times 6$ unbalanced lattice \\
\hline Amsterdam, MPU ${ }^{\mathrm{d}}$ & $20 / 02 / 01$ & -26.5728 & 30.72778 & 1478 & 881 & 14.8 & 700 & 26 & $5 \times 5$ unbalanced lattice \\
\hline Lothair, MPU & $22 / 02 / 01$ & -26.4833 & 30.63333 & 1600 & 869 & 14.6 & 800 & 25 & $5 \times 5$ triple lattice \\
\hline
\end{tabular}

In addition to comparing improved with unimproved material, entries included seed orchard bulks comprising a mix of the same mother families originating from different seedling seed orchards, i.e. approximate half sibs, to determine if seed orchard plays a role in progeny performance. Common seed/mother trees ranged from eight to 15 families, depending on bulk composition, the low number of common pollen parents allowing for potential variation between bulks to be expressed. All bulks from a specific seedling seed orchard were also combined in another comparison, irrespective of flowering level, to further examine the relationship between seed orchard and genetic gain. In order to establish whether there was a relationship between the number of trees flowering simultaneously in a seed orchard and progeny performance (i.e. assuming increased outcrossing with increased flowering, above a certain level of flowering), entries were included that comprised bulks of the same families, but which were collected in different years to represent different levels of flowering in the orchards. Flowering assessments were made in these orchards over three years to acquire the necessary flowering figures, which were obtained 
by totaling the number of flowering trees in a seed orchard and calculating these as a percentage of all trees in the orchard. Lastly, bulks comprising different family combinations were included to determine whether this played a significant role in achieved gain being commercially deployed. Flowering over the period fell between 15 and $20 \%$ or 40 and $47 \%$, and was thus categorised into these two levels $(\leq 20 \%$ and $\geq 40 \%$, respectively) for the purposes of this study. Details of the treatment/ bulk compositions, selection intensity and grouped comparisons are included in Tables $\mathbf{2}$ and $\mathbf{3}$.

Table 2 Individual entry comparisons in E. nitens genetic gain trials at three sites

\begin{tabular}{|c|c|c|c|c|}
\hline Entry no. & $\begin{array}{l}\text { Origin and year seed collected } \\
\text { (flowering percentage in previous year) }\end{array}$ & $\begin{array}{l}\text { Entry/b } \\
\text { composi }\end{array}$ & & Level of female selection \\
\hline $\begin{array}{l}1 \\
2\end{array}$ & $\begin{array}{l}\text { E88/01 Jessievale SO }{ }^{\mathrm{b}} \text { A } 1998(15 \%) \\
\text { E88/01 Jessievale SO A } 1999(40 \%)\end{array}$ & $\begin{array}{l}27832 \\
31328 \\
31329 \\
31331\end{array}$ & $\begin{array}{l}31332 \\
31337 \\
32101 \\
32098\end{array}$ & $\begin{array}{l}8 \text { top families from } 42 \\
8 \text { top families from } 42\end{array}$ \\
\hline $\begin{array}{c}3 \\
4 \\
9 \\
10\end{array}$ & $\begin{array}{l}\text { E88/03 Helvetia SO B } 2000(44 \%) \\
\text { E88/01 Jessievale SO B } 1998(15 \%) \\
\text { E88/01 Jessievale SO B } 2000(45 \%) \\
\text { E88/05 Jaglust SO B } 2000 \text { bulk }(47 \%)\end{array}$ & $\begin{array}{l}32079 \\
32087 \\
32089 \\
32090\end{array}$ & $\begin{array}{l}32093 \\
32095 \\
32097 \\
32100\end{array}$ & $\begin{array}{l}8 \text { top families from } 49 \\
8 \text { top families from } 42 \\
8 \text { top families from } 42 \\
8 \text { top families from } 144\end{array}$ \\
\hline 5 & E88/01 Amsterdam SO C 1998 (20\%) & \multicolumn{2}{|c|}{ Top $70 \%$ families } & 25 families from 34 \\
\hline $\begin{array}{l}6 \\
7\end{array}$ & $\begin{array}{l}\text { E88/05 Jaglust SO D } 1998 \text { bulk (47\%) } \\
\text { E88/01 Jessievale SO D } 1998 \text { bulk (15\%) }\end{array}$ & $\begin{array}{l}27832 \\
31331 \\
31338 \\
32084 \\
32087 \\
32091 \\
32092 \\
32094\end{array}$ & $\begin{array}{l}32095 \\
32096 \\
32097 \\
32099 \\
32100 \\
32101 \\
32102\end{array}$ & $\begin{array}{l}15 \text { top families from } 144 \\
15 \text { top families from } 42\end{array}$ \\
\hline 8 & E88/03 Helvetia SO E 2000 (44\%) & $\begin{array}{l}32087 \\
32093 \\
32095 \\
32096 \\
32100 \\
34831 \\
34832\end{array}$ & $\begin{array}{l}34833 \\
34835 \\
34836 \\
34837 \\
34838 \\
34839 \\
34840\end{array}$ & 16 top families from 49 \\
\hline $\begin{array}{l}11 \\
12 \\
13 \\
14 \\
16 \\
17 \\
18 \\
19 \\
20 \\
21\end{array}$ & $\begin{array}{l}\text { E88/01 Jessievale SO 1998, top family } \\
\text { E88/01 Jessievale SO 2000, top family } \\
\text { E88/03 Helvetia SO } 1999 \text {, top family } \\
\text { E88/03 Helvetia SO 2000, top family } \\
\text { E88/05 Jaglust SO } 1998 \text {, top family } \\
\text { E88/03 Helvetia SO 2000, top family } \\
\text { E88/05 Jaglust SO 1998, top family } \\
\text { E88/05 Jaglust SO 1998, top family } \\
\text { Land race commercial bulk, ex Dorstbult SO, SA } \\
\text { Improved commercial bulk, ex Helvetia SO, SA }\end{array}$ & $\begin{array}{l}32 \\
32 \\
32 \\
32 \\
34 \\
34 \\
37 \\
37\end{array}$ & $\begin{array}{l}7 \\
77 \\
7 \\
7 \\
32 \\
32 \\
32 \\
24\end{array}$ & \\
\hline 22 & Unimproved general bulk ex Australia & $\begin{array}{l}32083 \\
32091 \\
32092 \\
32093 \\
32096\end{array}$ & $\begin{array}{l}32099 \\
32101 \\
34832 \\
34838 \\
37628\end{array}$ & 10 families \\
\hline $\begin{array}{l}23 \\
24 \\
26 \\
27 \\
28 \\
29 \\
30\end{array}$ & $\begin{array}{l}\text { Unimproved average family ex Nelshoogte, SA } \\
\text { Unimproved top family ex Badja, Australia } \\
\text { Unimproved top family ex Barren Mountain, Australia } \\
\text { Unimproved top family ex Barrington Tops, Australia } \\
\text { Unimproved local bulk E. nitens ex Perdestal, SA, } 1989 \\
\text { E. grandis } x \text { nitens }(G X N) \text { clone ex SA } \\
\text { Controlled pollination seed ex SA }\end{array}$ & $\begin{array}{l}37 \\
32 \\
34\end{array}$ & $\begin{array}{l}32 \\
32 \\
32\end{array}$ & \\
\hline
\end{tabular}

\footnotetext{
${ }^{\mathrm{a}}$ See text for level of male selection $\quad{ }^{\mathrm{b}}$ Seedling seed orchard ${ }^{\mathrm{c}}$ South Africa
} 
Table 3 Combination of entries for group comparisons in E. nitens genetic gain trials at three sites

\begin{tabular}{llll}
\hline Group comparison & Entries included & Group comparison & Entries included \\
\hline i) Level of improvement & & iv) Seed Orchard & 5 \\
Unimproved & $20,21,22,28$ & Amsterdam & $3,8,13,14$ \\
Improved & $1-14$ & Helvetia & 6,10 \\
ii) Flowering level & & Jaglust & $1,2,4,7,9,11,12$ \\
$\leq 20 \%$ & $1,4,5,7$ & Jessievale & \\
$\geq 40 \%$ & $2,3,6,8,9,10$ & & 1,2 \\
iii) Year of seed collection & $1,4,5,6,7,11$ & A Composition of Seed Orchard Bulk \\
1998 & 2,13 & B & $3,4,9,10$ \\
1999 & $3,8,9,10,12,14$ & C & 5 \\
2000 & & D & 6,7 \\
\hline
\end{tabular}

\section{Measurements}

Diameter at breast height (dbh) and tree height measurements were carried out at Lothair and Amsterdam at 87 months after establishment and at Balgowan at 97 months, which is just prior to full rotation for eucalypts grown on a pulp rotation in the temperate areas of South Africa. Formal stem form and disease assessments were not carried out because these traits were bred to the desired level in the first-generation trials (Swain et al. 1998). Individual-tree volume was calculated from these measurements using the equation developed by Schnöau (1982):

$\log V=b_{0}+b_{1} \log (D+$ vald $)+b_{2} \log H$

where $V=$ total volume to $5 \mathrm{~cm}$ tip diameter in cubic decimetre, $D=\mathrm{dbh}$ in centimetre, $H=$ total height in metre, $b_{0}=-2.17055, \quad b_{1}=2.07516$, vald $=$ constant tree form value $=0$, and $b_{2}=1.42792$. The assumption of constant tree form value throughout is satisfactory (Bredenkamp 2000). Total treatment/entry volumes per plot were calculated and then estimated per hectare, taking survival into account.

\section{Statistical analysis}

Statistical analysis was conducted using SAS ${ }^{\circledR}$ Institute, Inc., Software 9.2 (SAS 2002-2008). Dead or missing trees were removed from the dataset before analysis. To test for normality for dbh, height and volume, residuals were plotted against fitted values. None showed any detectable trends or patterns and it can therefore be said that the condition $\varepsilon_{i j k} \sim$ iid $\left(0, \sigma^{2}\right)$ were met for these data, and the standard ANOVA assumptions are valid. Analyses of variance for dbh, height, survival, individual-tree and total volumes were carried out for each site, as well as across sites, and $F$ statistics were calculated to test for significant differences among entries. Proc GLM was used to calculate least squares means for dbh, height, survival, individual-tree and total volumes of each entry, as this procedure is recommended for unbalanced designs (Hettasch et al. 2007). Comparisons were made for differences between individual entries using Fisher's test for Least Significance Differences (LSD) at the $1 \%$ significance level. Simple and partial phenotypic correlation statistics were estimated between traits using the combined site entry means.

In addition, individual entries were grouped, and statistical comparisons were made for levels of improvement, flowering levels, year of seed collection, seedling seed orchard origin and composition of seedling seed orchard bulk for all traits, across sites. Comparisons within the entry groups were made using pairwise $t$ tests. The following model was used for the individual site, nine tree square plot analysis: 
$y_{i j k l}=\mu+\operatorname{rep}_{i}+\operatorname{block}_{j}\left(\operatorname{rep}_{i}\right)+\operatorname{tmt}_{k}+\operatorname{rep}_{i} * \operatorname{tmt}_{k}+\left(\operatorname{rep}_{i} * \operatorname{tmt}_{k}\right)+\varepsilon_{i j k l}$

where $y_{i j k l}=$ mean for the trait of the $l^{\text {th }}$ tree in the $i^{\text {th }}$ rep and $k^{\text {th }}$ entry, $\mu=$ overall mean, rep $=i^{\text {th }}$ rep effect (fixed), $i=1, \ldots, 4$; block $=j^{\text {th }}$ block within $i^{\text {th }}$ rep effect (fixed), $j=1, \ldots, 5 ;$ tmt $=k^{\text {th }}$ entry effect (random) $k=1, \ldots, 14,16, \ldots, 25,26$ or 29 ; rep*tmt $=$ interaction between the $i^{\text {th }}$ rep and $k^{\text {th }}$ entry (random plot effect); $\varepsilon_{i j k l}=$ random error associated with $i^{\text {th }}$ rep, $j^{\text {th }}$ block within $i^{\text {th }}$ rep, $k^{\text {th }}$ entry and $l^{\text {th }}$ tree where $\varepsilon_{i j k l} \sim$ iid $\left(0, \sigma^{2}\right)$.

The following model was used for the combined site analysis:

$y_{i j k l}=\mu+\operatorname{site}_{i}+\operatorname{rep}_{j}\left(\operatorname{site}_{i}\right)+\operatorname{tmt}_{k}+\left(\operatorname{site}_{l} * \mathrm{tmt}_{k}\right)+\varepsilon_{i j k l}$

where $y_{i j k l}=$ mean for the trait of the $l^{\text {th }}$ tree in the $j^{\text {th }}$ rep and $k^{\text {th }}$ entry at the $i^{\text {th }}$ site; $\mu=$ overall mean; site $=$ site effect (fixed), $i=1, \ldots, 3 ; \operatorname{rep}_{\mathrm{j}}\left(\operatorname{site}_{j}\right)=j^{\text {th }}$ rep effect (fixed) within $i^{\text {th }}$ site, $j=1, \ldots, 4 ; \mathrm{tmt}=k^{\text {th }}$ entry effect (random), $k=1, \ldots, 14,16, \ldots, 25,26$ or 29 ; site*tmt $=$ interaction between the $i^{\text {th }}$ site and $k^{\text {th }}$ entry (random); $\varepsilon_{i j k l}=$ random error associated with $i^{\text {th }}$ site, $j^{\text {th }}$ rep within $i^{\text {th }}$ site, $k^{\text {th }}$ entry and $l^{\text {th }}$ tree where $\varepsilon_{i j k l} \sim$ iid $\left(0, \sigma^{2}\right)$. Interactions between the grouped entries (i.e. level of improvement, flowering level, year of seed collection, seed orchard and bulk composition) were tested, and a regression analysis was performed on flowering levels with growth traits.

\section{RESULTS AND DISCUSSION}

\section{Comparison of individual entries}

Growth

Table 4 presents the final survival, individual-tree and total volume entry means for the three genetic gain trials. There were significant differences $(p<0.01)$ between entries for all traits at all three sites, and within-site replicate effects were significant for all traits except total volume. Block-within-replicate effects were not significant $(p>0.01)$ and replicate $\mathrm{x}$ entry interaction effects were only significant $(p<0.01)$ at Amsterdam for individual volume (details not shown). With regard to the combined site analysis, site effects were significant $(p<0.001)$ for all traits, but site $\mathrm{x}$ entry effects were only significant for total volume $(p<0.001)$ and survival $(p<0.05)$ (Table 5). Table 4 also presents the across or combined site survival, individual and total volume entry means. Across all sites, top performing entries 11 (top improved family 32097 from Jessievale seed orchard) and 17 (top improved family 34832 from Helvetia seed orchard) significantly outperformed $(p<0.05)$ the majority of unimproved entries, the land-race commercial bulk (entry 20), the $G X N$ hybrid clone (entry 29) and the two first-generation top families from Jaglust (entries 18 and 19), for total volume. Entry 27 (unimproved top family 34832 ex Barrington Tops, Australia) was only present at two sites, but performed well overall, being the top entry in the combined site analysis for total volume. At first glance, this would seem to indicate that the more northernmost Australian provenance of Barrington Tops should be widely used in future breeding. Although this is supported by results of first-generation trials in South Africa (Swain et al. 2013), the northern provenance of Barren Mountain performed as well as Barrington Tops in the first-generation trials.

With regards to poor performance, unimproved control entry 28 (unimproved South African E. nitens ex Perdestal) performed significantly worse $(p<0.01)$ than the majority of improved entries for most traits. 
Table 4 Final percentage survival, total volume and individual-tree volume entry means, ranked for decreasing total volume, in three $E$. nitens genetic gain trials, and a combined site analysis.

\begin{tabular}{|c|c|c|c|c|c|c|c|c|c|c|c|c|c|c|c|}
\hline \multirow[b]{2}{*}{ Entry } & \multicolumn{3}{|c|}{ Balgowan (97 months) } & \multicolumn{4}{|c|}{ Amsterdam (87 months) } & \multicolumn{4}{|c|}{ Lothair (87 months) } & \multicolumn{4}{|c|}{ Combined site analysis } \\
\hline & $\begin{array}{c}\text { Survival } \\
(\%)\end{array}$ & $\begin{array}{c}\text { Total } \\
\text { volume } \\
\left(\mathrm{m}^{3} \mathrm{ha}^{-1}\right) \\
(p<0.05)\end{array}$ & $\begin{array}{l}\text { Indiv. tree } \\
\text { volume }^{\mathrm{a}} \\
\left(\mathrm{m}^{3}\right)\end{array}$ & Entry & $\begin{array}{c}\text { Survival } \\
(\%)\end{array}$ & $\begin{array}{c}\text { Total } \\
\text { volume } \\
\left(\mathbf{m}^{3} \mathbf{h a}^{-1}\right) \\
(p<0.05)\end{array}$ & $\begin{array}{l}\text { Indiv. tree } \\
\text { volumea } \\
\left(\mathbf{m}^{3}\right)\end{array}$ & Entry & $\begin{array}{c}\text { Survival } \\
(\%)\end{array}$ & $\begin{array}{c}\text { Total } \\
\text { volume } \\
\left(\mathbf{m}^{3} \mathbf{h a}^{-1}\right)\end{array}$ & $\begin{array}{c}\text { Indiv. tree } \\
\text { volume } \\
\left(\mathbf{m}^{\mathbf{3}}\right)\end{array}$ & Entry & $\begin{array}{c}\text { Survival } \\
(\%)\end{array}$ & $\begin{array}{c}\text { Total } \\
\text { volume } \\
\left(\mathbf{m}^{3} \mathbf{h a}^{-1}\right) \\
(p<0.001)\end{array}$ & $\begin{array}{c}\text { Indiv. tree } \\
\text { volume }^{\mathrm{a}} \\
\left(\mathbf{m}^{3}\right)\end{array}$ \\
\hline 17 & 93 a & $404.8 \mathrm{a}$ & $0.262 \mathrm{a}$ & 17 & $63 \mathrm{bc}$ & $189.3 \mathrm{a}$ & $0.183 \mathrm{~b}$ & 10 & $85 \mathrm{ab}$ & $240.2 \mathrm{a}$ & $0.164 \mathrm{ab}$ & 27 & $89 \mathrm{a}$ & $257.6 \mathrm{a}$ & $0.174 \mathrm{ab}$ \\
\hline 11 & $83 \mathrm{ab}$ & $366.4 \mathrm{ab}$ & $0.264 \mathrm{a}$ & 13 & $75 \mathrm{a}$ & $189.2 \mathrm{a}$ & $0.151 \mathrm{~b}$ & 5 & $81 \mathrm{ab}$ & $236.5 \mathrm{a}$ & $0.082 \mathrm{~b}$ & 11 & $79 \mathrm{ab}$ & $252.5 \mathrm{a}$ & $0.195 \mathrm{ab}$ \\
\hline 2 & $86 \mathrm{ab}$ & $331.2 \mathrm{ab}$ & $0.231 \mathrm{ab}$ & 8 & $72 \mathrm{ab}$ & $182.5 \mathrm{a}$ & $0.152 \mathrm{~b}$ & 27 & $83 \mathrm{a}$ & $230.9 \mathrm{a}$ & $0.080 \mathrm{~b}$ & 8 & $79 \mathrm{ab}$ & 249.7 a & $0.195 \mathrm{ab}$ \\
\hline 6 & $81 \mathrm{ab}$ & $322.7 \mathrm{ab}$ & $0.240 \mathrm{ab}$ & 1 & $78 \mathrm{a}$ & $179.1 \mathrm{ab}$ & $0.136 \mathrm{~b}$ & 2 & $86 \mathrm{a}$ & $226.2 \mathrm{a}$ & $0.157 \mathrm{abc}$ & 17 & 70 abcde & $241.7 \mathrm{ab}$ & $0.206 \mathrm{a}$ \\
\hline 8 & $78 \mathrm{ab}$ & $321.9 \mathrm{ab}$ & $0.241 \mathrm{ab}$ & 11 & $67 \mathrm{~b}$ & $173.0 \mathrm{abc}$ & $0.156 \mathrm{~b}$ & 11 & $83 \mathrm{a}$ & $218.1 \mathrm{a}$ & $0.157 \mathrm{abc}$ & 2 & $76 \mathrm{abc}$ & $241.0 \mathrm{ab}$ & $0.186 \mathrm{ab}$ \\
\hline 14 & $72 \mathrm{ab}$ & $308.7 \mathrm{ab}$ & $0.256 \mathrm{a}$ & 14 & $69 \mathrm{ab}$ & $169.8 \mathrm{abc}$ & $0.142 \mathrm{~b}$ & 8 & $78 \mathrm{bc}$ & $209.9 \mathrm{ab}$ & $0.158 \mathrm{abc}$ & 5 & $73 \mathrm{abcd}$ & $226.6 \mathrm{ab}$ & $0.191 \mathrm{ab}$ \\
\hline 9 & $89 \mathrm{ab}$ & $307.1 \mathrm{ab}$ & $0.207 \mathrm{ab}$ & 2 & $61 \mathrm{bc}$ & $165.6 \mathrm{abc}$ & $0.160 \mathrm{~b}$ & 1 & $86 \mathrm{a}$ & $206.0 \mathrm{ab}$ & $0.144 \mathrm{bc}$ & 3 & 67 abcde & $222.2 \mathrm{ab}$ & $0.178 \mathrm{ab}$ \\
\hline 5 & $72 \mathrm{ab}$ & $296.0 \mathrm{ab}$ & $0.246 \mathrm{ab}$ & 3 & $75 \mathrm{a}$ & $160.2 \mathrm{abcd}$ & $0.127 \mathrm{~b}$ & 12 & $81 \mathrm{ab}$ & $199.8 \mathrm{ab}$ & $0.149 \mathrm{bc}$ & 14 & 69 abcde & $219.7 \mathrm{ab}$ & $0.185 \mathrm{ab}$ \\
\hline 4 & $75 \mathrm{ab}$ & $290.6 \mathrm{ab}$ & $0.233 \mathrm{ab}$ & 9 & $58 \mathrm{bc}$ & $155.5 \mathrm{abcd}$ & $0.159 \mathrm{~b}$ & 9 & $72 \mathrm{bc}$ & $191.0 \mathrm{ab}$ & $0.156 \mathrm{abc}$ & 6 & 74 abcd & $218.8 \mathrm{ab}$ & $0.175 \mathrm{ab}$ \\
\hline 1 & $82 \mathrm{ab}$ & $285.5 \mathrm{ab}$ & $0.211 \mathrm{ab}$ & 6 & $64 \mathrm{bc}$ & $153.7 \mathrm{abcd}$ & $0.144 \mathrm{~b}$ & 17 & $64 \mathrm{bc}$ & $184.8 \mathrm{ab}$ & $0.172 \mathrm{ab}$ & 1 & $81 \mathrm{a}$ & $217.9 \mathrm{ab}$ & $0.160 a b c$ \\
\hline 27 & $95 \mathrm{a}$ & $284.3 \mathrm{ab}$ & $0.181 \mathrm{ab}$ & 29 & $31 \mathrm{bc}$ & 148.1 abcde & $0.290 \mathrm{a}$ & 14 & $72 \mathrm{bc}$ & $180.5 \mathrm{ab}$ & $0.149 \mathrm{bc}$ & 9 & $73 \mathrm{abcd}$ & $217.9 \mathrm{ab}$ & $0.179 \mathrm{ab}$ \\
\hline 3 & $75 \mathrm{ab}$ & $284.1 \mathrm{ab}$ & $0.227 \mathrm{ab}$ & 5 & $61 \mathrm{bc}$ & 147.2 abcde & $0.144 \mathrm{~b}$ & 6 & $81 \mathrm{ab}$ & $180.0 \mathrm{ab}$ & $0.133 \mathrm{bc}$ & 13 & 68 abcde & $216.4 \mathrm{ab}$ & $0.183 \mathrm{ab}$ \\
\hline 16 & $64 \mathrm{ab}$ & $281.5 \mathrm{ab}$ & $0.264 \mathrm{a}$ & 10 & $61 \mathrm{bc}$ & 131.7 abcde & $0.128 \mathrm{~b}$ & 21 & $69 \mathrm{bc}$ & $178.2 \mathrm{ab}$ & $0.154 \mathrm{bc}$ & 10 & 72 abcde & $213.2 \mathrm{ab}$ & $0.181 \mathrm{ab}$ \\
\hline 12 & $78 \mathrm{ab}$ & $280.3 \mathrm{ab}$ & $0.216 \mathrm{ab}$ & 26 & $47 \mathrm{bc}$ & 122.8 abcde & $0.154 \mathrm{~b}$ & 7 & $75 \mathrm{bc}$ & $177.6 \mathrm{ab}$ & $0.145 \mathrm{bc}$ & 30 & $78 \mathrm{abc}$ & $200.9 \mathrm{abc}$ & $0.155 a b c$ \\
\hline 10 & $70 \mathrm{ab}$ & $274.4 \mathrm{ab}$ & $0.237 \mathrm{ab}$ & 12 & $44 \mathrm{bc}$ & 112.3 abcde & $0.159 \mathrm{~b}$ & 20 & $78 \mathrm{bc}$ & $172.8 \mathrm{ab}$ & $0.142 \mathrm{bc}$ & 12 & 70 abcde & $197.5 \mathrm{abc}$ & $0.175 \mathrm{ab}$ \\
\hline 30 & $78 \mathrm{ab}$ & $262.8 \mathrm{ab}$ & $0.203 \mathrm{ab}$ & 21 & $50 \mathrm{bc}$ & 109.6 abcde & $0.133 \mathrm{~b}$ & 30 & $78 \mathrm{bc}$ & $169.9 \mathrm{ab}$ & $0.130 \mathrm{bc}$ & 7 & 73 abcde & $181.9 \mathrm{abc}$ & $0.144 \mathrm{abc}$ \\
\hline 7 & $76 \mathrm{ab}$ & $248.7 \mathrm{ab}$ & $0.182 \mathrm{ab}$ & 24 & $44 \mathrm{bc}$ & 105.3 abcde & $0.150 \mathrm{~b}$ & 29 & $42 \mathrm{c}$ & $155.9 \mathrm{ab}$ & $0.222 \mathrm{a}$ & 4 & 61 abcde & $179.8 \mathrm{abc}$ & $0.166 \mathrm{abc}$ \\
\hline 13 & $67 \mathrm{ab}$ & $243.5 \mathrm{ab}$ & $0.219 \mathrm{ab}$ & 4 & $67 \mathrm{~b}$ & 103.2 abcde & $0.092 \mathrm{~b}$ & 28 & $75 \mathrm{bc}$ & $155.2 \mathrm{ab}$ & $0.121 \mathrm{bc}$ & 21 & 65 abcde & $174.8 \mathrm{abc}$ & $0.163 \mathrm{abc}$ \\
\hline 21 & $70 \mathrm{ab}$ & $215.9 \mathrm{ab}$ & $0.184 \mathrm{ab}$ & 7 & $67 \mathrm{~b}$ & 102.6 abcde & $0.094 \mathrm{~b}$ & 22 & $75 \mathrm{bc}$ & $149.1 \mathrm{ab}$ & $0.118 \mathrm{bc}$ & 16 & $58 \mathrm{bcde}$ & $164.5 \mathrm{abc}$ & $0.178 \mathrm{ab}$ \\
\hline 20 & $58 \mathrm{ab}$ & $210.2 \mathrm{ab}$ & $0.246 \mathrm{ab}$ & 20 & $56 \mathrm{bc}$ & 95.7 abcde & $0.106 \mathrm{~b}$ & 4 & $53 \mathrm{bc}$ & $145.6 \mathrm{ab}$ & $0.171 \mathrm{ab}$ & 20 & $57 \mathrm{cde}$ & $158.7 \mathrm{abc}$ & $0.154 \mathrm{abc}$ \\
\hline 18 & $75 \mathrm{ab}$ & $209.3 \mathrm{ab}$ & $0.168 \mathrm{ab}$ & 22 & $36 \mathrm{~cd}$ & 93.9 abcde & $0.155 \mathrm{~b}$ & 16 & $58 \mathrm{bc}$ & $129.5 \mathrm{ab}$ & $0.132 \mathrm{bc}$ & 22 & 62 abcde & $144.3 \mathrm{abc}$ & $0.141 \mathrm{abc}$ \\
\hline 23 & $67 \mathrm{ab}$ & $189.8 \mathrm{ab}$ & $0.171 \mathrm{ab}$ & 18 & $51 \mathrm{bc}$ & 93.6 abcde & $0.106 \mathrm{~b}$ & 24 & $50 \mathrm{bc}$ & $129.2 \mathrm{ab}$ & $0.162 \mathrm{abc}$ & 24 & $49 \mathrm{e}$ & $132.6 \mathrm{abc}$ & $0.151 \mathrm{abc}$ \\
\hline 19 & $70 \mathrm{ab}$ & $188.8 \mathrm{ab}$ & $0.163 \mathrm{ab}$ & 16 & $44 \mathrm{bc}$ & 82.4 bcde & $0.107 \mathrm{~b}$ & 26 & $72 \mathrm{bc}$ & $118.7 \mathrm{ab}$ & $0.107 \mathrm{bc}$ & 29 & $51 \mathrm{e}$ & $130.8 \mathrm{abc}$ & $0.157 \mathrm{abc}$ \\
\hline 22 & $67 \mathrm{ab}$ & $177.3 \mathrm{ab}$ & $0.160 \mathrm{ab}$ & 19 & $33 \mathrm{~d}$ & $71.6 \mathrm{cde}$ & $0.129 \mathrm{~b}$ & 23 & $50 \mathrm{bc}$ & $106.5 \mathrm{ab}$ & $0.120 \mathrm{bc}$ & 19 & 58 bcde & $130.2 \mathrm{abc}$ & $0.152 \mathrm{abc}$ \\
\hline 24 & $64 \mathrm{ab}$ & $163.4 \mathrm{ab}$ & $0.153 \mathrm{ab}$ & 23 & $39 \mathrm{~cd}$ & $61.8 \mathrm{de}$ & $0.103 \mathrm{~b}$ & 18 & $39 \mathrm{c}$ & $61.7 \mathrm{~b}$ & $0.094 \mathrm{c}$ & 18 & $54 \mathrm{de}$ & $121.5 \mathrm{bc}$ & $0.133 \mathrm{bc}$ \\
\hline 26 & $47 \mathrm{c}$ & $108.4 \mathrm{ab}$ & $0.138 \mathrm{ab}$ & 28 & $36 \mathrm{~cd}$ & $49.5 \mathrm{e}$ & $0.083 \mathrm{~b}$ & & & & & 26 & $54 \mathrm{de}$ & $116.2 \mathrm{bc}$ & $0.134 \mathrm{bc}$ \\
\hline 29 & $72 \mathrm{ab}$ & $73.8 \mathrm{ab}$ & $0.061 \mathrm{~b}$ & & & & & & & & & 23 & $50 \mathrm{e}$ & $114.9 \mathrm{bc}$ & $0.137 \mathrm{abc}$ \\
\hline 28 & $30 \mathrm{c}$ & $38.4 \mathrm{~b}$ & $0.078 \mathrm{ab}$ & & & & & & & & & 28 & $47 \mathrm{e}$ & $84.9 \mathrm{c}$ & $0.105 \mathrm{c}$ \\
\hline Trial mean & 72.1 & 248.1 & 0.205 & & 55.7 & 128.6 & 0.139 & & 69.8 & 173.5 & 0.149 & & 65.7 & 185.2 & 0.168 \\
\hline & 17.43 & 188.74 & 0.153 & & 18.88 & 54.19 & 0.090 & & 21.49 & 53.25 & 0.091 & & 19.64 & 74.07 & 0.121 \\
\hline
\end{tabular}

Values followed by the same letter of the alphabet within a column are not significantly different from each other $(p>0.01$, unless indicated otherwise)

${ }^{\mathrm{a}}$ Individual-tree volume $\quad{ }^{\mathrm{b}}$ Standard deviation of entry means 
Table 5 Analysis of variance for combined site percentage survival and growth, as well as growth within entry groups for the three genetic gain trials

\begin{tabular}{|c|c|c|c|c|c|c|c|}
\hline & Trait & $\begin{array}{c}\text { Source of } \\
\text { variation }\end{array}$ & $d f$ & $\begin{array}{c}\text { Mean } \\
\text { Square }\end{array}$ & $F$ value & $p$ value & \\
\hline \multirow{10}{*}{$\begin{array}{l}\text { Percentage } \\
\text { survival }\end{array}$} & \multirow{5}{*}{ Entry } & Site & 2 & 8196.743 & 21.27 & $<0.0001$ & *** \\
\hline & & Rep (site) & 9 & 611.339 & 1.59 & 0.12 & \\
\hline & & Entry & 27 & 1165.615 & 3.02 & $<0.0001$ & $* * *$ \\
\hline & & Site*entry & 49 & 390.207 & 1.01 & 0.46 & \\
\hline & & Error & 213 & 385.403 & & & \\
\hline & \multirow{5}{*}{$\begin{array}{l}\text { Level of } \\
\text { improvement }\end{array}$} & Site & 2 & 4751.905 & 16.07 & $<0.0001$ & $* * *$ \\
\hline & & Rep (site) & 9 & 283.383 & 0.96 & 0.48 & \\
\hline & & Improvement & 1 & 7330.620 & 24.78 & $<0.0001$ & $* * *$ \\
\hline & & Site*improvement & 2 & 1445.573 & 4.89 & 0.01 & $* *$ \\
\hline & & Error & 166 & 295.771 & & & \\
\hline \multirow{26}{*}{$\begin{array}{l}\text { Total } \\
\text { volume }\end{array}$} & \multirow{5}{*}{ Entry } & Site & 2 & 354315.929 & 69.96 & $<0.0001$ & $* * *$ \\
\hline & & Rep (site) & 9 & 5559.452 & 1.10 & 0.37 & \\
\hline & & Entry & 27 & 25933.600 & 5.12 & $<0.0001$ & $* * *$ \\
\hline & & Site*entry & 49 & 7318.113 & 1.44 & 0.04 & $*$ \\
\hline & & Error & 213 & 5064.653 & & & \\
\hline & \multirow{5}{*}{$\begin{array}{l}\text { Level of } \\
\text { improvement }\end{array}$} & Site & 2 & 62611.3621 & 12.44 & $<.0001$ & $* * *$ \\
\hline & & Rep (site) & 9 & 5708.6565 & 1.13 & 0.34 & \\
\hline & & Improvement & 1 & 207367.1799 & 41.19 & $<.0001$ & $* * *$ \\
\hline & & Site*improvement & 2 & 29193.6384 & 5.80 & 0.004 & $* *$ \\
\hline & & Error & 166 & 5034.029 & & & \\
\hline & \multirow{4}{*}{$\begin{array}{l}\text { Flowering level } \\
(\text { grouped } \leq 20 \text { and } \\
\geq 40 \%)\end{array}$} & Site & 2 & 225973.664 & 56.22 & $<0.0001$ & $* * *$ \\
\hline & & Rep (site) & 9 & 6466.717 & 1.61 & 0.13 & \\
\hline & & Flower & 1 & 16351.056 & 4.07 & 0.05 & $*$ \\
\hline & & Error & 102 & 4019.636 & & & \\
\hline & \multirow{4}{*}{$\begin{array}{l}\text { Year of seed } \\
\text { collection }\end{array}$} & Site & 2 & 305192.646 & 62.28 & $<0.0001$ & $* * *$ \\
\hline & & Rep (site) & 9 & 4718.353 & 0.96 & 0.48 & \\
\hline & & Year & 2 & 1929.537 & 0.39 & 0.68 & \\
\hline & & Error & 145 & 4900.134 & & & \\
\hline & \multirow{4}{*}{ Seed orchard } & Site & 2 & 304054.905 & 61.64 & $<0.0001$ & $* * *$ \\
\hline & & Rep (site) & 9 & 4735.810 & 0.96 & 0.48 & \\
\hline & & Seed orchard & 3 & 1350.865 & 0.27 & 0.84 & \\
\hline & & Error & 144 & 4932.819 & & & \\
\hline & \multirow{4}{*}{ Composition of bulk } & Site & 2 & 227622.635 & 57.42 & $<0.0001$ & $* * *$ \\
\hline & & Rep (site) & 9 & 6999.527 & 1.77 & 0.08 & \\
\hline & & Bulk & 4 & 8479.672 & 2.14 & 0.08 & \\
\hline & & Error & 99 & 3963.992 & & & \\
\hline \multirow{17}{*}{$\begin{array}{l}\text { Individual } \\
\text { tree } \\
\text { volume }\end{array}$} & \multirow{5}{*}{ Entry } & Site & 2 & 0.114 & 8.05 & 0.0006 & $* * *$ \\
\hline & & Rep (site) & 9 & 0.010 & 0.74 & 0.67 & \\
\hline & & Entry & 27 & 0.015 & 1.10 & 0.36 & \\
\hline & & Site*entry & 45 & 0.016 & 1.10 & 0.35 & \\
\hline & & Error & 102 & 0.014 & & & \\
\hline & \multirow{4}{*}{$\begin{array}{l}\text { Level of } \\
\text { improvement }\end{array}$} & Site & 2 & 0.823 & 56.85 & $<0.0001$ & **** \\
\hline & & Rep (site) & 9 & 0.051 & 3.57 & 0.0002 & $* * *$ \\
\hline & & Improvement & 1 & 0.240 & 16.58 & $<.0001$ & $* * *$ \\
\hline & & Error & 1145 & 0.014 & & & \\
\hline & \multirow{4}{*}{$\begin{array}{l}\text { Flowering level } \\
\text { (grouped } \leq 20 \text { and } \\
\geq 40 \%)\end{array}$} & Site & 2 & 0.596 & 42.90 & $<0.0001$ & $* * *$ \\
\hline & & Rep (site) & 9 & 0.033 & 2.38 & 0.01 & $*$ \\
\hline & & Flower & 1 & 0.030 & 2.14 & 0.14 & \\
\hline & & Error & 751 & 0.014 & & & \\
\hline & \multirow{4}{*}{$\begin{array}{l}\text { Year of seed } \\
\text { collection }\end{array}$} & Site & 2 & 0.844 & 57.55 & $<0.0001$ & $* * *$ \\
\hline & & Rep (site) & 9 & 0.043 & 2.96 & 0.0017 & $* *$ \\
\hline & & Year & 2 & 0.008 & 0.51 & 0.60 & \\
\hline & & Error & 1035 & 0.015 & & & \\
\hline
\end{tabular}




\begin{tabular}{|c|c|c|c|c|c|c|}
\hline \multirow{5}{*}{ Seed orchard } & Site & 2 & 0.091 & 7.31 & 0.0012 & $* *$ \\
\hline & Rep (site) & 9 & 0.018 & 1.48 & 0.17 & \\
\hline & Seed orchard & 3 & 0.012 & 0.97 & 0.41 & \\
\hline & Site*seed orch. & 6 & 0.033 & 2.68 & 0.02 & $*$ \\
\hline & Error & 88 & 0.012 & & & \\
\hline \multirow{4}{*}{ Composition of bulk } & Site & 2 & 0.604 & 43.60 & $<0.0001$ & $* * *$ \\
\hline & Rep (site) & 9 & 0.035 & 2.49 & 0.0082 & $*$ \\
\hline & Bulk & 4 & 0.024 & 1.73 & 0.14 & \\
\hline & Error & 748 & 0.014 & & & \\
\hline
\end{tabular}

$d f$ degrees of freedom

The control-pollinated seed (entry 30) performed at or below the trial mean at the two sites where it was established. This performance may have been relatively poor, either because the seed was produced from early control-pollinated crosses, where the technique was still being established and the levels of contamination may have been high, or due to poor specific combining ability of the genotypes.

\section{Survival}

It is notable that the ranking of many of the entries changed markedly once survival was taken into account, i.e. total volume per hectare was calculated with dead trees having a volume of zero. Survival differences were significant at varying levels at the individual sites, i.e. Balgowan $(p<0.05)$, Amsterdam $(p<0.005)$ and Lothair $(p<0.1)$, with no significant entry x replicate effects $(p>0.1)$ for the three sites (details not shown). In the combined site analysis, site effect was significant for survival $(p<0.001)$; yet the site $\mathrm{x}$ entry effect was non-significant $(p>0.05)$ (Table 5). With the exception of entry 27 (unimproved top family 34832 ex Barrington Tops, Australia), survival or stocking of the improved entries was generally better than that of the unimproved and land-race material (Table 4). The GXN hybrid clone performed well below the trial average at Balgowan yet had good survival (72\%), and at Amsterdam, although survival was poor (31\%), individual-tree growth of surviving trees was good as trees captured the open space around them. In contrast, entry 27 performed well at the two sites where it was planted, this performance being due in part to final survival of 95 and $83 \%$, respectively. All other unimproved entries (22, 23, 24, 26 and 28) had lower survival than most improved entries and low total volume, as if survival itself was behaving as a genetic trait. The positive impact of survival was expected after selection, as the previous generation of improvement focused on selection of trees that (1) had improved pest and/or disease tolerance, (2) were able to capture the site better, as measured by superior growth, and (3) had good survival. Selection for these traits has apparently resulted in increased stocking contributing significantly to the gain achieved through tree improvement.

Simple correlations $(r)$ between survival, dbh and height, as well as partial correlations between survival and total volume, are presented in Table 6. These indicate that the correlation between survival and height $(r=0.77)$ is greater than that of survival with dbh $(r=0.65)$, and that there is a positive correlation between $\mathrm{dbh}$ and height for this trial series $(r=0.74)$. This latter correlation is lower than that obtained in first-generation trials of E. nitens $(r \geq 0.82)$, the material being related to 
that included in this genetic gain trial series (Swain et al. 2013). With regard to the partial correlations of total volume with dbh and height (total volume being dependent on both dbh and height), the higher $r$ of 0.90 for total volume with height for constant dbh supported the stronger correlation between survival and height. Swain et al. (2013) present further trait and juvenile-mature correlations for the related first-generation material over a range of sites and trial series, as well as genetic parameters for the E. nitens population.

Table 6 Selected simple phenotypic correlations (below the diagonal) and partial correlations (above the diagonal) between traits for final measurements of the three genetic gain trials

\begin{tabular}{|c|c|c|c|c|}
\hline \multicolumn{5}{|c|}{ Pearson's phenotypic trait correlations $(p \leq 0.0002)$} \\
\hline Trait & dbh & Height & Survival & Total volume \\
\hline dbh & - & - & & \\
\hline Height & 0.74 & - & & \\
\hline Survival & 0.65 & 0.77 & - & $\begin{array}{l}0.78 \\
\text { (For constant height) } \\
0.90 \\
\text { (For constant dbh) }\end{array}$ \\
\hline Total volume & - & - & 0.91 & $-x^{2}$ \\
\hline
\end{tabular}

\section{Gains}

These results indicate that improvement has been made through the first generation of selection in the ICFR breeding programme, with the average increase in total volume of improved over unimproved material being $62.3 \%$ (Tables 4 and Table 7). Gains that can be made by using seed orchard bulks originating from any of the four ICFR seedling seed orchards included in these trials range from 9.3 to 94.4\% in total volume depending on site and bulk used, and expressed as a percentage of the unimproved and land-race bulk means, respectively (Shelbourne 1970). There were no significant differences $(p>0.01)$ between the improved bulks, although the bulk D from Jessievale (entry 7 (15\% flowering)) performed below the mean for all traits for the combined site analysis, and similarly, bulk B from Jessievale (entry 4 (15\% flowering)) performed just below the mean for total volume and individual-tree volume (Table 4). Both commercial bulks i.e. the improved commercial bulk from Helvetia (entry 21) and the land-race commercial bulk from Dorstbult (entry 20), were average performers for dbh but were below the mean for volumes and height in the combined site analysis (Table 4). As there were no significant differences between the different E. nitens seed orchard bulks in this study, nor the individual top-performing families, the homogeneity of the various entries was investigated. This showed that the range of dbh was similar for all entries across all sites, in the range of 15 to $20 \mathrm{~cm}$, with only two exception: $G X N$ (entry 29) had a narrower range of variation of $9 \mathrm{~cm}$, as would be expected from a clone, and the unimproved South African bulk from Perdestal (entry 28) had a narrow range in the lower dbh range. Although the literature provides many comparisons between unimproved and improved eucalypt seedlots, most of which show significant improvements of the bred material over the unimproved material, very few comparisons have been found that displayed significant differences between improved eucalypt open-pollinated seed orchard bulks of the same species and nominal level of improvement. This is supported by findings in previous E. macarthurii 
(Swain et al. 1999) and E. nitens genetic gain trials (Jones, pers. comm ${ }^{1}$ ) in South Africa and in E. camaldulensis genetic gain trials in India (Varghese et al. 2009), where bulks of the same nominal level of improvement did not differ significantly from each other.

Although there were no significant differences $(p>0.01)$ between the improved seed orchard bulks, the yield improvement of these bulks over the unimproved controls varied markedly according to which bulk was used in the comparison. The improved commercial bulk E from Helvetia (entry 8, $44 \%$ flowering) produced an average of 91.0 and $164.8 \mathrm{~m}^{3} \mathrm{ha}^{-1}$ more than the land-race commercial bulk from Dorstbult (entry 20) and the unimproved South African bulk from Perdestal (entry 28), respectively. By contrast, the bulk D from Jessievale (entry 7, $15 \%$ flowering) produced only 23.2 and $97.0 \mathrm{~m}^{3} \mathrm{ha}^{-1}$ more than the two controls, respectively.

\section{Comparison of grouped entries}

Tables 5 and 7 present comparisons of grouped entries for different levels of improvement, flowering, year of seed collection, seedling seed orchard and seed orchard bulk composition.

Table 7 Comparison of growth within entry groups in E. nitens genetic gain trials across all sites

\begin{tabular}{|c|c|c|c|c|c|}
\hline & Grouping & $\begin{array}{l}\text { Total volume } \\
\left(\mathrm{m}^{3} \mathrm{ha}^{-1}\right)\end{array}$ & $\begin{array}{l}\text { dbh } \\
(\mathrm{cm})\end{array}$ & $\begin{array}{l}\text { Height } \\
\text { (m) }\end{array}$ & $\begin{array}{c}\text { Individual-tree } \\
\text { volume } \\
\left(\mathrm{m}^{3} \text { tree }^{-1}\right)\end{array}$ \\
\hline \multirow{2}{*}{ Level of improvement } & Improved & $217.95 \mathrm{a}$ & $15.26 \mathrm{a}$ & $20.59 \mathrm{a}$ & $0.178 \mathrm{a}$ \\
\hline & Unimproved & $114.61 \mathrm{~b}$ & $13.60 \mathrm{~b}$ & $18.33 \mathrm{~b}$ & $0.125 \mathrm{~b}$ \\
\hline \multirow{2}{*}{ Flowering percentage } & $\geq 40 \%$ & $227.62 \mathrm{a}$ & $15.50 \mathrm{a}$ & $20.77 \mathrm{a}$ & $0.183 \mathrm{a}$ \\
\hline & $\leq 20 \%$ & $200.79 \mathrm{~b}$ & $14.81 \mathrm{~b}$ & $20.20 \mathrm{~b}$ & $0.115 b$ \\
\hline \multirow{3}{*}{ Year of seed collection ${ }^{a}$} & 1999 & $231.14 \mathrm{a}$ & $15.51 \mathrm{a}$ & $20.83 \mathrm{a}$ & $0.185 \mathrm{a}$ \\
\hline & 2000 & $219.98 \mathrm{a}$ & $15.40 \mathrm{a}$ & $20.56 \mathrm{a}$ & $0.183 \mathrm{a}$ \\
\hline & 1998 & $212.41 \mathrm{a}$ & $15.06 \mathrm{a}$ & $20.55 \mathrm{a}$ & $0.172 \mathrm{a}$ \\
\hline \multirow{4}{*}{ Seed orchard } & Helvetia & $228.51 \mathrm{a}$ & $15.60 \mathrm{a}$ & $20.59 \mathrm{a}$ & $0.187 \mathrm{a}$ \\
\hline & Amsterdam & $226.57 \mathrm{a}$ & $15.77 \mathrm{a}$ & $20.60 \mathrm{a}$ & $0.190 \mathrm{a}$ \\
\hline & Jaglust & $216.11 \mathrm{a}$ & $15.25 \mathrm{a}$ & $20.74 \mathrm{a}$ & $0.178 \mathrm{a}$ \\
\hline & Jessievale & $212.20 \mathrm{a}$ & $15.03 \mathrm{a}$ & $20.55 \mathrm{a}$ & $0.172 \mathrm{a}$ \\
\hline \multirow{5}{*}{$\begin{array}{l}\text { Composition of seed } \\
\text { orchard bulk }^{\text {b }}\end{array}$} & $\mathrm{E}$ & $249.69 \mathrm{a}$ & $15.78 \mathrm{a}$ & $20.48 \mathrm{a}$ & $0.195 \mathrm{a}$ \\
\hline & $\mathrm{C}$ & $226.57 \mathrm{ab}$ & $15.78 \mathrm{a}$ & $20.61 \mathrm{a}$ & $0.191 \mathrm{ab}$ \\
\hline & A & $229.95 \mathrm{ab}$ & $15.19 \mathrm{a}$ & $20.63 \mathrm{a}$ & $0.173 \mathrm{ab}$ \\
\hline & $\mathrm{B}$ & $206.84 \mathrm{bc}$ & $15.23 \mathrm{a}$ & $20.60 \mathrm{a}$ & $0.176 \mathrm{ab}$ \\
\hline & $\mathrm{D}$ & $199.60 \mathrm{bc}$ & $14.66 \mathrm{a}$ & $20.33 \mathrm{a}$ & $0.159 \mathrm{~b}$ \\
\hline
\end{tabular}

Values within an entry grouping within a column followed by the same letter of the alphabet are not significantly different from each other $(p>0.05)$

a Refer to Table 2 for details of flowering in these years

${ }^{\mathrm{b}}$ Refer to Table 2 for details of bulk composition

\footnotetext{
${ }^{1}$ Jones W (2010) Shaw Research Centre, Tweedie, PO Box 473, Howick, 3290, SOUTH AFRICA.
} 


\section{Levels of improvement}

There were significant differences $(p<0.01)$ between the level of improvement for all traits (supporting the findings in Table 4).

\section{Flowering level}

Significant differences $(p<0.01)$ were found between flowering levels for all traits, with seed collected from seed orchards that had $\geq 40 \%$ flowering producing progeny with significantly greater volume than seed that was collected from seed orchards with $\leq 20 \%$ flowering (Table 7). It is unlikely that survival in the parent seed orchards would have affected flowering percentage, as there was good representation of the top $70 \%$ of families in the seed orchards, despite subsequent poor flowering in a few of the orchards some years. Mining of the survival data of the progeny for the different flowering levels did not show any consistent resultant high or low survival for the $\geq 40 \%$ or $\leq 20 \%$ flowering entries, respectively, as these seemed to differ across site and with flowering level (Table 4).

There is little research on the breeding system of E. nitens, but Moncur et al. (1995) estimated a 75\% outcrossing rate in this species, and Pound et al. (2003) found that levels of self-incompatibility in E. nitens ranged from 25.8 to $93.6 \%$. Self-pollination is definitely possible in E. nitens (Griffin et al. 1987; Tibbits 1988), particularly in areas where the presence of natural pollinators is low, and pollen load is poor. This is despite selfing being controlled by a late-acting self-incompatibility system where ovule abortions occur after self-pollination (Pound et al. 2003), and resultant inbreeding depression has been reported in nine-year-old trees originating from controlled self-pollinations of E. nitens (Hardner and Tibbits 1998).

A regression analysis performed on the complete range of flowering levels for the four different growth traits indicated a slight significant positive trend $(p<0.1)$ between increasing levels of flowering and progeny tree growth for all traits except total volume. However, the $R^{2}$ values were very low for all traits, indicating a poor fit of the model, and no conclusions can be drawn from this analysis. A comparison of percentage improvement, as determined by flowering level, showed the following improvements in total volume over the $15 \%$ flowering level: $40 \%$ flowering $(25.4 \%), 45 \%$ flowering (20.1\%), $20 \%$ flowering (17.9\%) and $47 \%$ flowering (12.0\%). The flowering levels happened to be specific to the design of each of the seed orchards that seed was collected from both in terms of family and final spacial distribution of parent trees; i.e. these were trials thinned to seed orchards based on family and individual performance and were not originally planted as seed orchards. This may partly explain the inconsistency of gain related to flowering level. Although a decrease in outcrossing rates has been linked to a decrease in progeny growth in forestry species (E. nitens, Hardner and Tibbits 1998; Eucalyptus globulus, Hardner and Potts 1995; Patterson et al. 2004; Acacia mangium, Butcher et al. 2004; Harwood et al. 2004), the flowering levels in this study do not necessarily represent the rate of outcrossing in the seed orchards, although the trends appear to be similar. Consequently, it could be assumed that an increase in flowering above a certain low level may result in increased gains in a 
population due to an increase in outcrossing rate, a decrease in selfing and subsequent inbreeding depression, but that additional flowering above this level may confer very little, if any benefit.

\section{Year of seed collection}

The year of seed collection did not differ significantly ( $p>0.1$ ) (Tables 5 and 7).

\section{Bulk composition}

The composition of the seed orchard bulks differed in that bulk E performed significantly better $(p<0.1)$ than bulks D and B for total volume, and better than bulk D for individual-tree volume (Tables 5 and 7). Bulk E comprised a mix of 14 top-performing families where seed was collected in a year following $\geq 40 \%$ flowering. By contrast, the poorer performing bulk D comprised two entries of 15 families representing the top $40 \%$ of families during years of $\leq 20 \%$ and $\geq 40 \%$ flowering in two different seed orchards, respectively. Bulk B comprised seven top and one average family in years following $\leq 20 \%$ and $\geq 40 \%$ flowering in three different seed orchards. Although this might imply that flowering level was influencing the bulk performance, bulk E did not perform significantly better than other bulks with low flowering levels, i.e. bulk A (15 and $40 \%$, bulk D $20 \%$ ).

\section{Seed orchard}

There were no significant differences $(p>0.1)$ in progeny growth based on seedling seed orchard. As not all seed orchards were represented by both high and low levels of flowering, which may have been biasing the data, the $\leq 20 \%$ flowering levels were removed from a subsequent analysis so that only the higher flowering levels were represented in all seed orchards. This had no effect on significance, with seed orchard still showing no impact on progeny growth.

This could imply that, irrespective of flowering levels in these seed orchards, seed can be utilised from any of these four seed orchards to achieve the same appreciable level of gain and production in commercial plantations. This is similar to what was found in Pinus taeda (Sluder 1988). Unfortunately there were insufficient degrees of freedom for the seed orchard $\mathrm{x}$ flowering level interaction to be tested in the current study, which may have further informed this. Although the seed orchard $\mathrm{x}$ bulk interaction was not significant $(p>0.05)$ for dbh or volume, certain combinations of flowering level, bulk composition and seedling seed orchard resulted in marked differences in progeny growth, as discussed earlier (Table 4). Caution should thus be exercised when compiling bulks from seedling seed orchards with low flowering levels in any given year. It may be necessary to ensure that certain maternal families that produce high-yielding progeny are included in these, or all, seedling seed orchard bulks.

To this end, a study on the mating system of this population of E. nitens should be carried out to determine how many individuals or families are involved in pollination in these E. nitens seed orchards, the levels of outcrossing and how much self-incompatibility varies with genotype. This will add to an understanding of the degree of selfing and outcrossing which is occurring in the seed 
orchards and the effect on the genetic quality of the seed.

\section{CONCLUSIONS}

Significant improvements have been made over the first generation of selection in the ICFR E. nitens breeding population. It is therefore recommended that seed from any of the ICFR improved bulks be accessed for commercial deployment when available, rather than using unimproved or land-race material from Australia and South Africa, respectively.

Improvement in survival of the advanced-generation material plays an important role in the gains in total volume per hectare achieved. In addition, indications are that levels of flowering have an impact on progeny growth. These results suggest that seed orchards with $15 \%$ flowering result in poorer progeny growth than those with $\geq 40 \%$ flowering, although this is not consistent and it is thus difficult to draw any definite conclusions in this regard. Indications are that flowering above a certain low level may result in increased gains in a population due to a decrease in selfing or related crosses, but that additional flowering above this level may confer very little, if any benefit. Further investigation of flowering levels should be carried out with larger numbers of observations per flowering level. Until then, it is recommended that seed should be collected, where possible, from seed orchards where $40 \%$ or more flowering was observed in the previous year. This is supported by substantial percentage improvement in total volume of the progeny, generally being more than $20 \%$ (and $p<0.05$ ) at these higher levels of flowering.

The orchard from which the seed is collected appears to have no effect on progeny growth in this trial series, irrespective of flowering levels. This suggests that seed collected from any of the four ICFR seedling seed orchards tested in the trial series will produce trees with significant improvement in growth over the unimproved and commercial material. It should however be noted that certain combinations of seedling seed orchard and bulk composition, particularly at the lower levels of flowering, produced much better progeny growth than others, even if this difference is not statistically significant. It is thus recommended that such higher yielding bulk and seedling seed orchard combinations be used for commercial deployment. This will impact on management of ICFR seed orchards and future seed bulk composition.

Molecular studies in the E. nitens seed orchards will provide a better understanding of selfing and outcrossing in this breeding population. This, in turn, will allow for manipulation of current and future seed orchards to ensure that maximum gains are captured in the seed for commercial deployment.

\section{Acknowledgements}

The foresters and research staff at Mondi Group, NCT Forestry Co-Operative Ltd. and Sappi Forests are gratefully acknowledged for their assistance with the establishment and maintenance of these trials. Thank you to the Technical Staff in the ICFR Tree Improvement Programme for the establishment and 
measurement of these trials, and to the reviewers of this document for their constructive comments. We are also grateful to the two reviewers and associate editor who made important contributions to the improvement of this manuscript.

\section{Conflict of interest}

The authors declare that the experiments described in this research paper comply with the current laws of South Africa and that there is no conflict of interest between authors.

\section{REFERENCES}

Bredenkamp B (2000) Volume and mass of logs and standing trees. In: Southern African Forestry Handbook 2000 Vol 1. The Southern African Institute of Forestry, Pretoria, South Africa, pp. 167-174

Butcher P, Harwood C, Quang TH (2004) Studies of mating systems in seed stands suggest possible causes of variable outcrossing ratesin natural populations of Acacia mangium. Forest Genetics 11(3-4): 303-309

Carlson C, Swain T-L, Soko S (2000) Preliminary investigation into nutritional differences between shy and early flowering families of Eucalyptus nitens. ICFR Bulletin Series 04/2000. Institute for Commercial Forestry Research, Pietermaritzburg, South Africa

Clarke CRE (2000) Wood and pulp properties of four New South Wales provenances of Eucalyptus nitens grown on a warm and a cold site in South Africa. Appita Journal 53 (3):231-236

Darrow WK (1994) Species trials of cold-tolerant eucalypts in the summer rainfall zone of South Africa. ICFR Bulletin Series 10/1994. Institute for Commercial Forestry Research, Pietermaritzburg, South Africa

Darrow WK (1996) Species trials of cold-tolerant eucalypts in the summer rainfall zone of South Africa: Results at six years of age. ICFR Bulletin Series 09/1996. Institute for Commercial Forestry Research, Pietermaritzburg, South Africa

de Little DW, Tibbits WN, Rasmussen GF, Ravenwood I (1992). Genetic improvement strategy for APPM Eucalypt tree farms in Tasmania. In: Mass Production Technology for Genetically Improved Fast Growing Forest Tree Species. ACTES proceedings, Bordeaux, 14-18 Septembre, 1992. AFOCEL (Association Foret Cellulose) Nangis, France, pp. 177-188

Eldridge K, Davidson J, Harwood C, van Wyk G (1993) Eucalypt Domestication and Breeding. Oxford University Press Inc., Oxford, U.K

Gardner RAW (2001) Site-species interaction studies with cold tolerant eucalypts at high altitudes in South Africa. In: Proceedings of IUFRO Working Group 2.08.03 Conference. Developing the Eucalypt of the Future. Valdivia, Chile, 10-15 September 2001

Gardner RAW (2003) Floral induction in Eucalyptus nitens (Deane \& Maiden) Maiden in South Africa. MSc Thesis, University of Natal, Pietermaritzburg, South Africa

Gardner RAW, Bertling I (2005) Effect of winter chilling and paclobutrazol on floral bud production in Eucalyptus nitens. South African Journal of Botany 71(2):238-249

Gardner RAW, Swain T-L (1996) Snow damage to timber plantations in KwaZulu-Natal during July 1996 and subsequent species recommendations. ICFR Bulletin Series 08/1996. Institute for Commercial Forestry Research, Pietermaritzburg, South Africa

Gardner RAW, Swain T-L, Norris C (2003) Eucalypt species and Provenance trials in the Southern Cape: Results at four years of age. ICFR Bulletin Series 11/2003. Institute for Commercial Forestry Research, Pietermaritzburg, South Africa

Griffin AR, Moran GF, Fripp YJ (1987) Preferential outcrossing in Eucalyptus regnans F. Muell. Australian Journal of Botany 35:465-475

Hardner CM, Potts BM (1995) Inbreeding depression and changes in variation after selfing Eucalyptus globulus subsp. globulus. Silvae Genetica 44:46-54

Hardner C, Tibbits W (1998) Inbreeding depression for growth, wood and fecundity traits in Eucalyptus nitens. Forest Genetics 51:11-20

Harwood CE, Thinh HH, Quang TH, Butcher PA and Williams ER (2004). The effect of inbreeding on early growth of Acacia mangium in Vietnam. Silvae Genetica 53(2):65-69

Hettasch MH, Snedden CL, Eatwell KA, Pierce BT, Verryn SD (2007) Practical Data Analysis for Tree 
Breeders Manual. Natural Resources and the Environment, CSIR, Pretoria, South Africa

Jones W R. 2002. Breeding Systems of Some Cold Tolerant Eucalyptus Species. MSc Thesis, University of Natal, Pietermaritzburg, South Africa

Kunz R, Gardner RAW (2001) A review of factors affecting snow damage of commercial forest plantations in South Africa. ICFR Bulletin Series 13/2001. Institute for Commercial Forestry Research, Pietermaritzburg, South Africa

Moncur MW (1998) Hastening seed production: a tool for increasing the rate of genetic improvement in eucalypt species. In: Tree Improvement: Applied Research and Technology Transfer. Ed: Puri S. Science Publishers, Inc., USA

Moncur MW, Mitchell A, Fripp Y, Kleinschmidt GJ (1995) The role of honey bees (Apis mellifera) in eucalypt and acacia seed production areas. Canadian Journal of Forest Research 24:46-49

Patterson B, Vaillancourt RE, Pilbeam DJ and Potts BM (2004) Factors affecting variation in outcrossing rate in Eucalyptus globulus. Australian Journal of Botany 52:773-780

Pound LM, Wallwork MAB, Potts BM, Sedgley M (2003) Pollen tube growth and early ovule development following self- and cross-pollination in Eucalyptus nitens. Sexual Plant Reproduction 16:59-69

SAS Institute (2002-2008) SAS/STAT Computer Software. Release 9.2. SAS Institute Inc. Cary, North Carolina, USA

Schönau APG (1982) Timber Volume and Utilization Tables for Six Common Eucalypts. Wattle Research Institute, Pietermaritzburg, South Africa. 64pp. ISBN 0869802828.

Sedgley M, Hand FC, Smith RM, Griffin AR (1989) Pollen tube growth and early seed development in Eucalyptus regnans F. Muell. (Myrtaceae) in relation to ovule structure and preferential outcrossing. Australian Journal of Botany 37:397-411

Shelbourne CJA (1970) Genetic improvement in different tree characteristics of Pinus radiata and the consequences for silviculture and utilisation. Pp 44-57. In: James, R N, Sutton W R J, Tustin J R (Comp.) Pruning and Thinning Practice. New Zealand Forest Science, FRI Symposium No. 12

Sluder ER (1988) Inhertiance and Gain in a Half-Diallel Cross Among Loblolly Pines Selected for Resistance to Fusiform Rust. Slivae Genetica 37(1):22-26

Swain T-L (2001) ICFR Tree Improvement Project: Status Report - Eucalyptus nitens. ICFR Bulletin Series 06/2001. Institute for Commercial Forestry Research, Pietermaritzburg, South Africa

Swain T-L, Chiappero CC (1998) Collection of improved E. nitens seed from ICFR seed orchards. ICFR Newsletter May 1998. Institute for Commercial Forestry Research, Pietermaritzburg, South Africa. Pp 7-10

Swain T-L, Gardner RAW (2003) A summary of current knowledge of cold tolerant eucalypt species (CTE's) grown in South Africa. ICFR Bulletin Series 03/2003. Institute for Commercial Forestry Research, Pietermaritzburg, South Africa

Swain T-L, Chiappero CC, Gardner RAW (1998) Final measurements of six ICFR E. nitens provenance/progeny trials in the summer rainfall region of South Africa. ICFR Bulletin Series 05/1998. Institute for Commercial Forestry Research, Pietermaritzburg, South Africa

Swain T-L, Verryn SD, Laing MD (2013) Genetic characterisation of a Eucalyptus nitens base breeding population in South Africa. Submitted December 2012 (in manuscript)

Tibbits WN (1998) Germination and morphology of progeny from controlled pollinations of Eucalyptus nitens (Deane \& Maiden) Maiden. Australian Journal of Botany 36:677-691

Varghese M, Kamalakannan R, Harwood CE, Lindgren D, McDonald MW (2009) Changes in growth performance and fecundity of Eucalyptus camaldulensis and E. tereticornis during domestication in southern India. Tree Genetics and Genomes (5):629-640 